\title{
Tratamento Clínico e Seguimento das Hiperplasias de Endométrio
}

\author{
Clinical Treatment and Follow-up of Endometrial Hyperplasia \\ Anaglória Pontes ${ }^{1}$, Paulo Traiman ${ }^{1}$, Marcello Franco ${ }^{2}$, Eliana Aguiar Petri Nahás ${ }^{1}$ \\ Rogério Dias ${ }^{1}$, Laurival Antonio De Luca ${ }^{1}$ \\ RESUMO
}

\begin{abstract}
Objetivos: avaliar a eficácia do acetato de medroxiprogesterona e do acetato de megestrol nas hiperplasias de endométrio.

Métodos: foram incluidas, retrospectivamente 47 pacientes com sangramento uterino anormal, submetidas a curetagem uterina diagnóstica e/ou biópsia de endométrio, cujo achado histopatológico foi de hiperplasia de endométrio. Nas pacientes com hiperplasia sem atipia foi iniciado a terapêutica com acetato de medroxiprogesterona por via oral, na dose de $10 \mathrm{mg} / \mathrm{dia}$ durante 10-12 dias por mês. Nas com atipia, era utilizado o acetato de megestrol por via oral, dose de $160 \mathrm{mg} /$ dia, uso continuo. O periodo de tratamento variou de 3 a 18 meses. Biópsia de endométrio e/ ou curetagem uterina de controle foram realizadas entre três e seis meses do inicio do tratamento e periodicamente para avaliar a resposta terapêutica.

Resultados: foram analisadas 42 pacientes com hiperplasia endometrial sem atipia e cinco com atipia. A média de idade das pacientes foi de 49,5 \pm 10,6 anos, sendo 70,2\% com idade superior a 45 anos. O acetato de medroxiprogesterona foi eficaz em fazer regredir as hiperplasias sem atipias em 83,2\% (35/42) e o acetato de megestrol em 80\% (4/5) das hiperplasias com atipia. Em 16,8\% (7 casos) das hiperplasias sem atipia e em 20\% (1 caso) das com atipia, ocorreu persistência das lesões, apesar do tratamento. Em nenhum caso ocorreu progressão para câncer de endométrio, durante o periodo de seguimento que foi de 3 meses a 9 anos. No acompanhamento dessas pacientes, verificamos que $18(38,3 \%)$ apresentaram amenorréia, em 12 (25,5\%) ocorreu regularização do ciclo menstrual e 17 (36,2\%) permaneceram com sangramento uterino anormal, sendo submetidas a histerectomia total abdominal. $O$ exame anatomopatológico mostrou a persistência da lesão hiperplásica em oito casos, leiomioma em quatro, adenomiose em três, mio-hipertrofia uterina difusa em um caso e útero normal em outro, tendo havido regressão das lesões hiperplásicas nesses últimos nove casos.

Conclusões: o tratamento das hiperplasias de endométrio com acetato de medroxiprogesterona e/ ou acetato de megestrol, representa uma alternativa satisfatória para mulheres que desejam preservar o útero ou que tenham risco cirúrgico elevado. Entretanto, é necessário monitorização cuidadosa do endométrio, o que deve ser realizado pela avaliação dos sintomas, ultra-sonografia transvaginal e biópsia periódica.
\end{abstract}

PALAVRAS-CHAVE: Hiperplasia de endométrio. Progestagenos. Sangramento uterino anormal. Endométrio: carcinoma.

\section{Introdução}

As hiperplasias de endométrio represen-

\footnotetext{
Departamento de Ginecologia e Obstetrícia da Faculdade de Medicina de Botucatu - UNESP

2 Departamento de Patologia da Faculdade de Medicina de Botucatu - UNESP

Correspondência:

Anaglória Pontes

Departamento de Ginecologia e Obstetrícia

Faculdade de Medicina de Botucatu - UNESP

18618-970 - Botucatu - SP

FAX: (14) 6822-1933 - Fone: (14) 6802-6227
}

tam um espectro de proliferação anormal, algumas constituindo precursores do carcinoma de endométrio. É definida como proliferação glandular, com aumento da relação glândula/ estroma, quando comparada ao endométrio proliferativo $^{1}$. Atualmente é classificada em duas categorias: hiperplasia sem e com atipia citológica; As hiperplasias podem ser simples ou complexas, levando-se em consideração anomalias citológicas e arquiteturais. Geralmente ocorrem de modo difuso, não necessariamente envolvendo todo o endométrio, podendo ainda 
ser focais ou multicêntricas.

Devido às várias classificações, à confusão na terminologia e à incerteza sobre o comportamento das diversas formas de hiperplasia, o diagnóstico e o tratamento nem sempre são adequados. No intuito de uniformizar a terminologia entre clínicos e patologistas, a "International Society of Gynecological Pathologists" e a "World Health Organization" (1994), baseadas nas características citológicas (típicas ou atípicas) e arquiteturais (simples ou complexas), adotaram a seguinte classificação para as hiperplasias do endométrio $^{1,2}$ : hiperplasia simples (cística sem atipia); hiperplasia complexa (adenomatosa sem atipia); hiperplasia simples com atipia (cística com atipia) e hiperplasia complexa com atipia (adenomatosa com atipia)

O termo hiperplasia adenomatosa tem sido empregada por diferentes autores, para descrever padrões histológicos distintos com ou sem atipia ${ }^{3,4}$. Gusberg et al, ${ }^{3}$, observaram que $18 \%$ das 562 pacientes com hiperplasia adenomatosa (o termo foi utilizado para descrever todos os tipos de hiperplasia exceto a hiperplasia cística) desenvolveram adenocarcinoma de endométrio dentro de um a três anos após o diagnóstico inicial.

Kurman et al. ${ }^{5}$, avaliaram retrospectivamente (média de 13,4 anos do diagnóstico) 170 pacientes com hiperplasia endometrial não-tratadas, que haviam sido submetidas a curetagem uterina. Verificaram que a progressão para carcinoma ocorreu em $1 \%$ das pacientes com hiperplasia simples sem atipia, em $3 \%$ das com hiperplasias complexas sem atipia, em $8 \%$ das com hiperplasia simples atipica e em $29 \%$ das com hiperplasia complexa atípica.

A partir da década de oitenta, o conceito de hiperplasia com ou sem atipia vem sendo modificado, considerado como duas entidades diferentes, para cuja classificação é de fundamental importância a presença de atipia citológica ${ }^{6}$. O risco da hiperplasia progredir para carcinoma de endométrio está relacionado à presença e à gravidade da atipia citológica, como também à complexidade e à estratificação arquitetural ${ }^{1}$.

Ferenczy et al. ${ }^{6}$ argumentam que a hiperplasia sem atipia em geral não é lesão precursora do carcinoma, ao passo que havendo atipias, correlaciona-se ao câncer de endométrio, e deveria ser denominada de neoplasia endometrial intraepitelial (NEI). A duração média de progressão de uma hiperplasia sem atipia é de aproximadamente 10 anos e com atipia é de quatro anos ${ }^{5}$.

As pacientes com hiperplasia de endométrio apresentam, como manisfetação clínica, sangramento uterino anormal, embora ocasionalmente estas lesões possam ser detectadas em mulheres assintomáticas ${ }^{1}$. As hiperplasias de endométrio desenvolvem-se como resultado de estí- mulo estrogênico sem oposição da progesterona e ocorrem com maior freqüência em pacientes anovuladoras crônicas, obesas, com doença hepática, que fizeram terapia de reposição hormonal com estrogênios sem progesterona ou com tumores ovarianos produtores de estrogênios ${ }^{2,7}$.

Wentz ${ }^{8}$ mostrou que o tratamento com dimetisterona (100 mg/dia) ou megestrol $(20 \mathrm{mg} /$ dia) por 6 semanas em 54 pacientes poderia controlar a hiperplasia atípica do endométrio por um período de pelo menos 3 anos. Entretanto, Eichner e Abellera ${ }^{9}$ verificaram que, embora o acetato de medroxiprogesterona $(80 \mathrm{mg} /$ dia) ou megestrol $(80 \mathrm{mg} /$ dia) provoquem regressão da hiperplasia durante a terapia, há recorrência ou progressão após a interrupção da medicação.

Gal et al. ${ }^{10}$ iniciaram o tratamento com acetato de megestrol (AM) (40 mg/dia), uso contínuo, em 52 pacientes na pós-menopausa com hiperplasia adenomatosa ou atípica ou carcinoma in situ de endométrio e que apresentavam risco cirúrgico elevado. Observaram, em acompanhamento de 10 a 104 meses (média de 42 meses), que mais de $90 \%$ destas mulheres apresentaram remissão completa da hiperplasia. Além do que, $90 \%$ dessas pacientes apresentavam obesidade mórbida, 65\% doença cardiovascular e $29 \%$ diabetes mellitus, além de outras doenças de risco para o tratamento cirúrgico.

Ferenczy e Gelfand ${ }^{11}$ realizaram estudo prospectivo em 85 mulheres menopausadas com hiperplasia de endométrio (65 sem atipia e 20 com atipia), seguidas por período médio de 7 anos (2 - 12 anos). Nas hiperplasias sem atipia utilizaram o acetato de medroxiprogesterona (AMP), por via oral na dose de $10 \mathrm{mg} /$ dia por 14 dias/mês, por 6 meses e nas com atipia, AMP na dose 20mg/dia, uso contínuo durante 6 meses. Encontraram regressão da hiperplasia sem atipia em $80 \%$ dos casos, persistência em $14 \%$ e em $6 \%$ houve recorrência após descontinuação da terapia; nenhuma paciente desenvolveu câncer. Por outro lado, nas pacientes com hiperplasia atípica, em $25 \%$ houve regressão, 25\% desenvolveram adenocarcinoma, em $25 \%$ houve recorrência e em $25 \%$ persistência.

No tratamento das hiperplasias endometriais é importante levar em consideração as características clínicas da paciente e identificar o tipo histológico. Na hiperplasia atípica, o tratamento de eleição é cirúrgico; só se utiliza o tratamento clínico nos casos de risco cirúrgico elevado ou quando as pacientes desejam preservar o útero. Os agentes progestacionais podem ser utilizados com reversão do quadro hiperplásico, na maioria dos $\operatorname{casos}^{10,11}$.

Os progestagênios são potentes antiestrogênicos quando utilizados em doses farmacológicas. Estimulam a atividade da $17 \beta$ - 
hidroxi esteróide desidrogenase e da sufonil transferase, que convertem o estradiol em sulfato de estrona, o qual é rapidamente excretado da célula. Diminuem os efeitos dos estrogênios sobre as células-alvo, pela inibição do aumento dos receptores estrogênicos, além de supressão da transcrição de oncogenes mediada pelos estrogênios, levando a efeito antimitótico e antiproliferativo sobre o endométrio ${ }^{12-14}$. Portanto, a terapia com acetato de medroxiprogesterona ou acetato de megestrol é alternativa clínica para o tratamento das pacientes com hiperplasia de endométrio que apresentam risco cirúrgico elevado ou que se recusam a se submeter a cirurgia, ou ainda em pacientes jovens que desejam concepção.

O objetivo deste trabalho foi avaliar a eficácia do acetato de medroxiprogesterona ou acetato de megestrol em pacientes com achado histopatológico de hiperplasia de endométrio.

\section{Pacientes e Métodos}

Foram incluídas 47 pacientes com sangramento uterino anormal tratadas entre 1988 e 1997. A avaliação dos casos foi retrospectiva com revisão do prontuário. Todas as pacientes cujo achado histopatológico foi de hiperplasia de endométrio foram submetidas a curetagem uterina (CTC) padrão sob anestesia e/ou biópsia de endométrio (BE) em nivel ambulatorial com cureta Novak. Estas pacientes foram acompanhadas no Ambulatório de Endométrio do Departamento de Ginecologia e Obstetrícia do Hospital das Clínicas da Faculdade de Medicina de Botucatu. O diagnóstico histopatológico foi realizado pelo Departamento de Patologia. Este trabalho obteve parecer favorável do Comitê de Ética e Pesquisa da referida Faculdade.

De acordo com a avaliação clínica e as características histopatológicas, e após explicação detalhada dos riscos, beneficios e contra-indicações, era oferecido a paciente o tratamento clínico ou cirúrgico. Se, apesar dos esclarecimentos, a paciente ainda assim desejasse preservar o útero ou apresentasse concomitantemente outra doença como obesidade mórbida, doença cardiovascular, diabetes mellitus, hipertensão arterial grave que tornasse o procedimento cirúrgico de risco elevado segundo os critérios da "American Society of Anesthesiologists" (ASA), era iniciada a terapêutica com acetato de medroxiprogesterona nas hiperplasias típicas e acetato de megestrol nas atípicas.

Foram excluídas deste estudo pacientes que faziam uso de terapia de reposição hormonal, que não tinham condições de fazer o seguimen- to ambulatorial, que não desejassem fazer o tratamento clínico ou que apresentassem contraindicação para o uso de progestagênios tais como tromboembolismo, hipercolesterolemia, depressão, infarto do miocardio, doença hepática.

Nos casos de hiperplasia sem atipia, simples ou complexa, foi introduzido o acetato de medroxiprogesterona (AMP) por via oral, na dose de $10 \mathrm{mg}$ ao dia durante 10-12 dias por mês, por um periodo inicial de três a seis meses. Após esse tempo, as pacientes eram submetidas a biópsia de endométrio de controle ou curetagem uterina diagnóstica. Se a hiperplasia persistisse, a dose de AMP era aumentada para 20 a $40 \mathrm{mg}$ por dia, por $12-14$ dias, por mais três ciclos. Após nova BE, se ainda assim persistisse a hiperplasia, era indicado a histerectomia. Se, com três a seis meses houvesse regressão da lesão (endométrio proliferativo, secretor, atrófico, menstrual, ou com decidualização do estroma), o tratamento era mantido por mais três meses; mantida a regressão, a paciente continuaria com AMP 5 a 10 $\mathrm{mg} /$ por 10 dias por mês, por 12 meses, quando era realizada $\mathrm{BE}$ de controle. A partir deste periodo, e de acordo com os sintomas e achados clínicos, era realizada $\mathrm{BE}$ ou ultra-sonografia transvaginal, para se avaliar a espessura endometrial e se esta era maior que $4 \mathrm{~mm}$ era realizada $\mathrm{BE}$ e/ou curetagem uterina de controle.

Nas pacientes com hiperplasia endometrial atípica, simples ou complexa, que tinham risco cirúrgico elevado, ou que recusavam submeterse a histerectomia, era utilizado o acetato de megestrol (AM) na dose de $160 \mathrm{mg}$ ao dia, de modo contínuo, por tempo indeterminado.

A BE e/ou CTG foram realizadas da mesma forma que nas hiperplasias típicas, isto é, dentro de três a seis meses do início do tratamento. Nesses casos, a ultra-sonografia e a BE eram realizadas a cada seis e doze meses para se avaliar a resposta do endométrio ao progestagênio. Após a introdução do AMP ou AM, a paciente era reavaliada quanto à recorrência do sangramento e efeitos colaterais.

A medicação era interrompida ou a dose diminuída, em face da intensidade dos seguintes efeitos colaterais decorrentes do uso dos progestogênios: depressão, retenção hídrica, náuseas, cólicas abdominais, irritabilidade, ansiedade, cefaléia e enxaqueca.

Foram analisados dados clínicos como sintomas, idade, menarca, paridade, padrão menstrual anterior e doenças associadas como obesidade, diabetes mellitus e hipertensão arterial.

A obesidade foi definida pelo índice de massa corpórea (IMC) de acordo com fórmula de Quetelet: peso $(\mathrm{kg})$ dividido pela estatura $(\mathrm{m})$ ao quadrado sendo o IMC considerado normal entre $18-25 \mathrm{~kg} / \mathrm{m}^{2}$, sobrepeso entre $25-30 \mathrm{~kg} / \mathrm{m}^{2}$, obesidade entre $30-40 \mathrm{~kg} / \mathrm{m}^{2}$, obesidade 
morbida $\geq 40 \mathrm{~kg} / \mathrm{m}^{215}$.

A hipertensão arterial foi diagnosticada, segundo os parâmetros clássicos de medida de pressão arterial em três oportunidades diferentes, estando a pressão arterial diastólica e/ou a sistólica constantemente elevadas, acima de $90 \mathrm{mmHg}$ e/ ou $160 \mathrm{mmHg}$ respectivamente, ou a paciente fazia uso de medicação anti-hipertensiva.

O diagnóstico de diabetes mellitus foi feito quando as pacientes apresentavam duas glicemias de jejum acima de $140 \mathrm{mg} / \mathrm{dl}$ ou ultimamente quando duas aferições após jejum de oito horas mostravam valores acima de $126 \mathrm{mg} / \mathrm{dl}^{16}$.

Foram realizadas periodicamente a glicemia de jejum, avaliações hepática, renal e perfil lipídico.

\section{Resultados}

Esse estudo incluiu um total de 47 pacientes, sendo que 42 apresentavam hiperplasia endometrial sem atipia, que representou $89,4 \%$ dos casos, e cinco com atipia $(10,6 \%)$, as quais fizeram uso de AMP e AM, respectivamente, por um período que variou de 3-18 meses.

A idade das pacientes variou de 22 a 72 anos com média de 49,5 $\pm 10,6$ anos, sendo que $70,2 \%$ (33/47) apresentavam idade superior a 45 anos (Figura 1). A menarca ocorreu entre 9 e 16 anos, com média de $12,8 \pm 1,5$; e a maioria das pacientes 88,9 (40/45) tinha antecedente de ciclos menstruais regulares e só $10,6 \%$ (5/47 casos) eram nuliparas. Em 16 casos (34\%), a queixa foi de sangramento pós-menopausa, $16(34 \%)$ metrorragia, $12(25,6 \%)$ hipermenorragia, dois $(4,3 \%)$ hipermenorréia e um $(2,1 \%)$ polimenorréia (Figura 2$)$.

O IMC mostrou que 55,5\% (25/45) das pacientes eram obesas (IMC $\geq 30$ ) (Figura 3). Das 47 pacientes com hiperplasia endometrial, 31,9\% (15/47), eram hipertensas crônicas e 23,4\% (11/ 47) apresentavam diabetes mellitus.

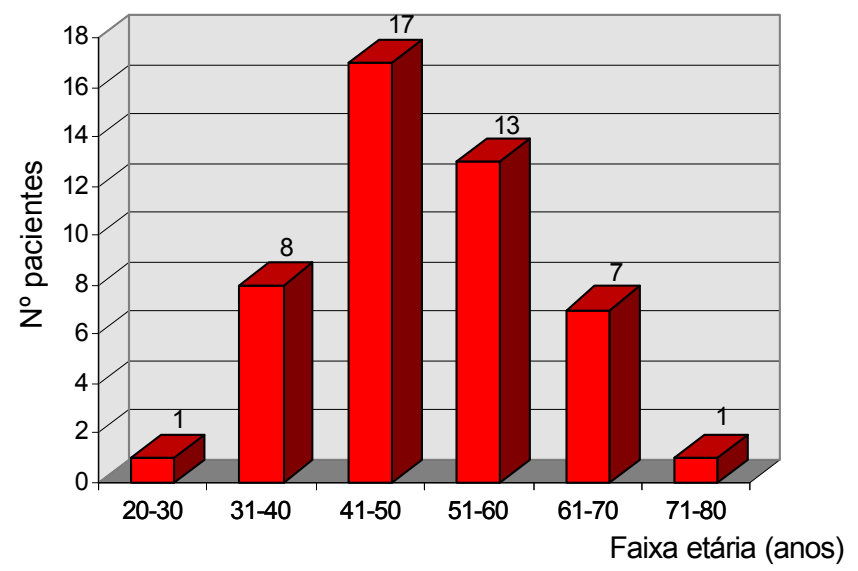

Figura 1 - Distribuição das 47 pacientes com hiperplasia de endométrio de acordo com a faixa etária.

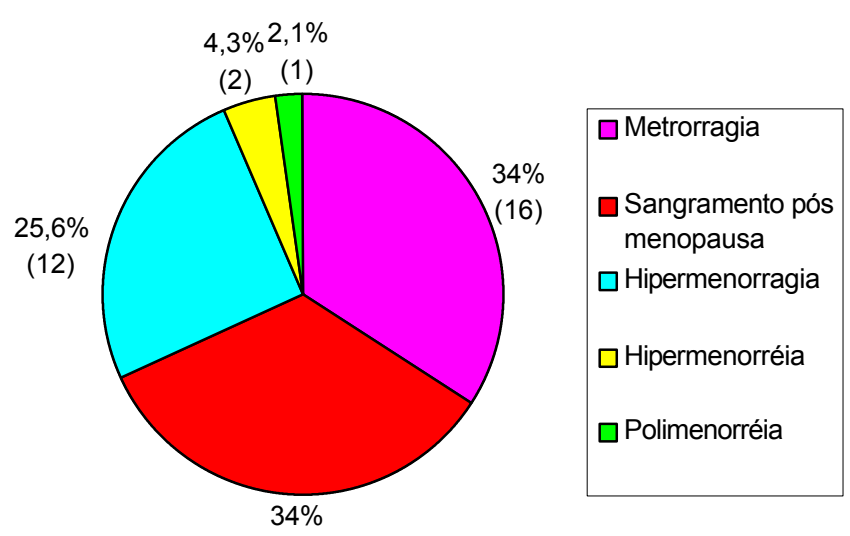

(16)

Figura 2 - Padrão de sangramento (\%) nas 47 pacientes com hiperplasia de endométrio
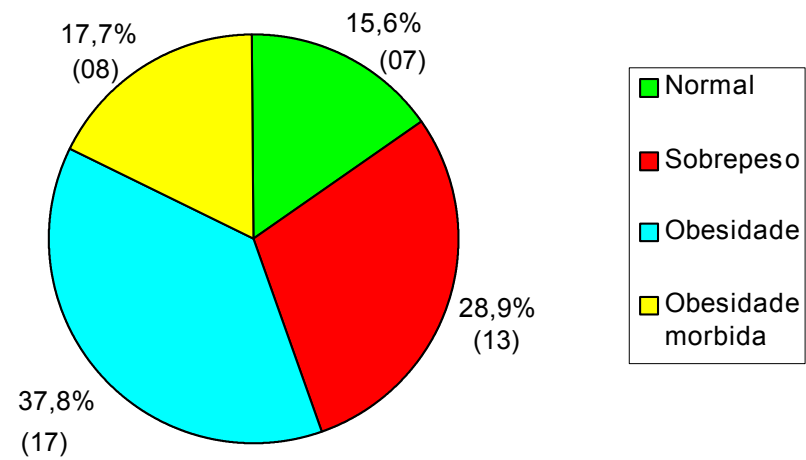

Figura 3 - Distribuição das pacientes com hiperplasia de endométrio de acordo com o Índice de Massa Corpórea (IMC): normal entre 18-25 kg/m²; sobrepeso entre $25-30 \mathrm{~kg} / \mathrm{m}^{2}$; obesidade entre $30-40 \mathrm{~kg} / \mathrm{m}^{2}$; obesidade mórbida 40 ou mais $\mathrm{kg} / \mathrm{m}^{2}$. Em 2 casos os dados não foram obtidos.

A classificação histopatológica das hiperplasias está apresentada na Figura 4 e a resposta ao uso do AMP e do AM na Tabela 1.

O AMP foi eficaz na regressão dos casos de hiperplasia sem atipia em $83,2 \%$ casos $(35 / 42)$; em $16,8 \%$ (7 casos), houve persistência e em nenhum caso ocorreu progressão para câncer de endométrio. É interessante observar que nesses sete casos, inicialmente houve regressão e, após interrupção do tratamento, observou-se recorrência da lesão hiperplásica. Na hiperplasia atípica, o AM foi eficaz em fazer regredir essa lesão em quatro casos (80\%), com persistência em uma paciente $(20 \%)$, a qual foi submetida a histerectomia. Como nos casos de hiperplasia sem atipia, não ocorreu progressão para câncer de endométrio (Tabela 1) durante o período de seguimento, que foi de três meses a nove anos.

No acompanhamento dessas pacientes, verificou-se que no decorrer do tratamento $38,3 \%$ (18) apresentaram amenorréia, em 25,5\% (12) ocorreu regularização do ciclo menstrual e $36,2 \%$ (17) permaneceram com sangramento uterino anormal (Figura 5), as quais foram submetidas a histerectomia total abdominal.

Algumas pacientes referiram cefaléia, tonturas, sonolência, cólicas abdominais e descon- 
forto pélvico e depressão durante o uso da medicação, mas que não impediram a continuação do tratamento.

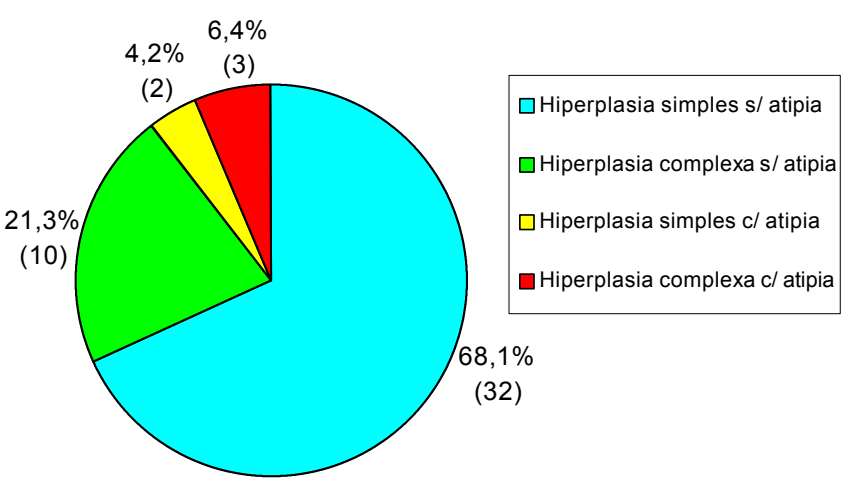

Figura 4-Distribuição das 47 pacientes com hiperplasia de endométrio de acordo com a classificação histopatológica.

Tabela 1 - Resposta ao tratamento com acetato de medroxiprogesterona, 10mg/dia, 10- 12 dias/ mês e acetado de megestrol $160 \mathrm{mg} / \mathrm{dia}$, uso contínuo, nas pacientes hiperplasia de endométrio sem atipia e com atipia.

\begin{tabular}{|c|c|c|c|c|c|c|}
\hline \multirow{2}{*}{$\begin{array}{l}\text { Resposta ao } \\
\text { tratamento* }\end{array}$} & \multicolumn{2}{|c|}{$\begin{array}{l}\text { Hiperplasia } \\
\text { sem atipia }\end{array}$} & \multicolumn{2}{|c|}{$\begin{array}{l}\text { Hiperplasia } \\
\text { com atipia }\end{array}$} & \multicolumn{2}{|c|}{ Total } \\
\hline & $n^{0}$ & $\%$ & $n^{0}$ & $\%$ & $n^{0}$ & $\%$ \\
\hline Regressão & 35 & 83,2 & 04 & 80,0 & 39 & 83,0 \\
\hline Persistência & 07 & 16,8 & 01 & 20,0 & 08 & 17,0 \\
\hline Total & 42 & 100 & 05 & 100 & 47 & 100 \\
\hline
\end{tabular}

* Nota: não houve casos com progressão para câncer de endométrio

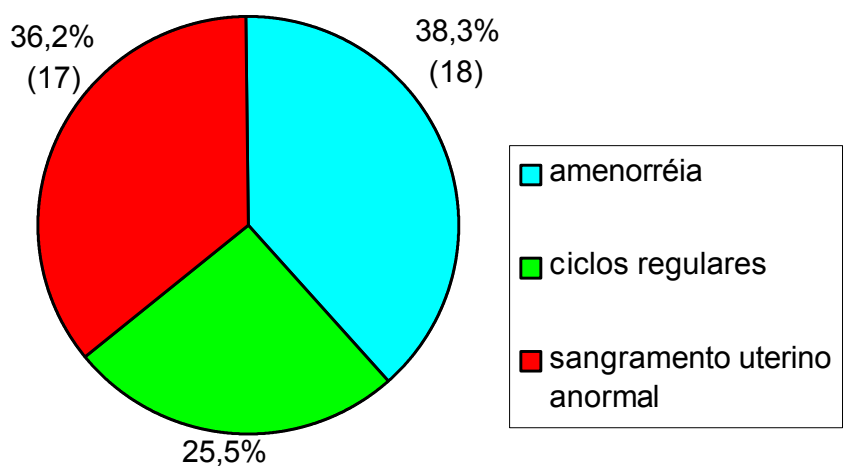

(12)

Figura 5 - Padrão menstrual (\%) das 47 pacientes com hiperplasia de endométrio, submetidas ao tratamento com acetato de medroxiprogesterona e acetato de megestrol.

\section{Discussão}

As hiperplasias de endométrio compreendem um grupo de alterações proliferativas, com potenciais biológicos diferentes, indo desde a forma de simples hiperplasia de caráter benigno até alterações histopatológicas acentuadas com atipia, as quais estão relacionadas com o câncer de endométrio ${ }^{11}$.

A conduta na hiperplasia de endométrio é indicada levando-se em consideração fatores clínicos associados às características histopatológicas, sendo a mais importante a presença de atipia citológica ${ }^{1,2}$.

Pacientes com hiperplasia simples ou complexa sem atipia podem ser tratadas clinicamente, pois o risco da progressão para câncer de endométrio é baixo, em torno de $1 \%$ a $3 \%$, ao passo que as mulheres com hiperplasia atípica têm risco elevado variando de $8 \%$ a $29 \%{ }^{5}$.

A hiperplasia endometrial atípica é lesão pré-cancerosa e a histerectomia é a terapêutica de escolha. Entretanto, algumas mulheres recusam-se submeter-se à histerectomia ou têm risco cirúrgico elevado, devido a problemas médicos como obesidade mórbida, doença cardiovascular, diabetes mellitus, hipertensão arterial, doença hepática ou renal que tornam o procedimento cirúrgico perigoso ${ }^{10}$.

Um dos mais interessantes avanços no tratamento das hiperplasias é a possibilidade de reversão das alterações hiperplásicas com o uso de agentes progestacionais ${ }^{10,11,17}$. Os progestagênios promovem, no endométrio, acentuada reação pseudodecidual do estroma e atrofia funcional das glândulas ${ }^{18}$. O AMP e o AM, progestagênios sintéticos derivados da 17-alfahidroxiprogesterona, são eficazes por via oral e podem ser usados no tratamento das hiperplasias de endométrio revertendo a maioria das lesões hiperplásicas ${ }^{10,11,19,20}$. O tratamento com progestagênios é entretanto prolongado e requer biópsias endometriais periódicas para confirmar a resolução das lesões ${ }^{21}$.

Os resultados deste trabalho mostram que a idade média de ocorrência da hiperplasia de endométrio foi de 49,5 $\pm 10,6$ anos, sendo que $70,2 \%$ tinham idade superior a 45 anos, dados esses compativeis com os da literatura, que afirmam que a hiperplasia de endométrio ocorre preferencialmente entre 45 e 55 anos de idade ${ }^{2}$.

$\mathrm{Na}$ avaliação clínica das pacientes, pode-se observar que com o uso do AMP e do AM, 30 das 47 pacientes, 63,8\%, apresentaram regularização do ciclo menstrual ou entraram em amenorréia. Recorrência ou persistência de sangramento uterino anormal ocorreu em 17 dos 47 casos, $32,6 \%$, sendo indicador clínico de que não houve regressão da lesão hiperplásica ou a paciente era portadora de outra doença uterina. A persistência do sangramento anormal fez com que estas mulheres reconsiderassem e aceitassem o tratamento cirúrgico. O exame anatomopatológico do útero desses 17 casos mostrou a persistência das lesões hiperplásicas em oito casos; nos outros nove, apesar da regressão da lesão hiperplásica, foi observada a presença de leiomioma em qua- 
tro, adenomiose em três, mio-hipertrofia uterina difusa em um e útero normal em outro caso.

O AMP e o AM foram eficazes em fazer regredir a hiperplasia na maioria dos casos, não ocorrendo em nenhum deles progressão para carcinoma de endométrio, o que coincide com os resultados de Gal et al. ${ }^{10}$, que relataram sucesso terapêutico com acetato de megestrol $40 \mathrm{mg} /$ dia, contínuo, no controle da hiperplasia endometrial em um grupo de pacientes com risco elevado para cirurgia. Apesar de retrospectivo, nossos resultados estão de acordo com o estudo prospectivo de Ferenczy e Gelfand ${ }^{11}$, que mostraram que o tratamento com AMP fez regredir a maioria das lesões hiperplásicas sem atipia e não aumentou o risco para o desenvolvimento de câncer endometrial. Em relação às hiperplasias atípicas, não foi observada progressão para cancer de endométrio em nenhum dos casos tratados com $160 \mathrm{mg}$ de $\mathrm{AM}$, ao passo que aqueles autores encontraram progressão para câncer em 25\% das pacientes com hiperplasia atípica tratadas com $20 \mathrm{mg}$ de AMP de modo contínuo.

A biópsia de endométrio periódica nas hiperplasias é aconselhável, pois apesar do tratamento, a lesão pode persistir, ou recidivar após interrupção do tratamento, como ocorreu em sete casos desse trabalho.

A hiperplasia de endométrio é considerada, algumas vezes evento transitório, de caráter benigno, principalmente no climatério, em que ocorre declínio da capacidade funcional do ovário, levando a ciclos anovulatórios e, conseqüentemente, proliferação exagerada do endométrio ${ }^{22}$. Esta afirmação está de acordo com o trabalho retrospectivo de Kurman et al. ${ }^{5}$, que verificaram que na maioria $(74 \%)$ das lesões hiperplásicas, ocorreu regressão espontânea, mesmo sem o tratamento. Também observamos regressão espontânea das hiperplasia sem atipia em sete pacientes (não incluídas neste estudo), que não foram tratadas com AMP e reavaliadas nove meses, 12 meses e entre quatro a nove anos após o diagnóstico inicial. A biópsia de endométrio de controle realizada em cinco e histerectomia em dois casos, mostrou remissão completa da lesão hiperplásica.

Trabalhos prospectivos recentes mostram que a hiperplasia endometrial sem atipia pode desaparecer espontaneamente em curto periodo de tempo de três meses ${ }^{23}$.

Condições que resultam na exposição endógena ou exógena a estrogênios, sem oposição da progesterona, como na obesidade, esteve presente em 55,5\% dos casos. Além disso, é interessante salientar que quatro (80\%) das cinco pacientes com hiperplasia atipica apresentavam obesidade mórbida (IMC $\geq 40$ )

$\mathrm{Na}$ mulher obesa, ocorre aumento da produção de estrona devido a três mecanismos: aumento da conversão periférica da androstenediona para estrona; diminuição na produção hepática da globulina carreadora dos hormônios sexuais (SHBG) e diferenças no metabolismo dos estrogênios com aumento do estrona livre circulante $^{21}$. Embora a estrona seja estrogênio fraco, ela é convertida para 17 $\beta$-estradiol (estrogênio potente) nas células endometriais. Os estrogênios induzem a proliferação e replicação das células endometriais, além de promoverem a formação de seus próprios receptores ${ }^{2,22}$; se os niveis estiverem elevados, pode ocorrer hiperplasia ${ }^{24}$. Por sua vez, a progesterona tem efeito oposto aos estrogênios no tecido endometrial levando à diminução do número de receptores estrogênicos, aumentando a conversão do $17 \beta$-estradiol para estrona, e diminuindo a sintese de RNA, induzida pelos estrogênios $^{2}$, reduzindo as lesões hiperplásicas.

Hoje, acredita-se que as hiperplasias de endométrio apresentam dois comportamentos biológicos distintos. Um, de caráter benigno, denominado hiperplasia que, na maioria das vezes, regride espontaneamente após a curetagem ou tratamento com progestagênios e que em geral, não é precursor do câncer do endométrio e representa uma resposta proliferativa exagerada em nivel endometrial. As lesões consideradas benignas incluem: endométrio proliferativo, anovulatório, hiperplasia glandular simples, cística ou adenomatosa sem atipia. O outro, de caráter neoplásico, é precursor do câncer de endométrio. Neste caso, estão incluidas todas as lesões hiperplásicas com atipia citonuclear ${ }^{5,6,11}$.

Os dados obtidos neste trabalho sugerem que a maioria das mulheres com hiperplasia endometrial respondem ao tratamento com AMP ou com AM, e a não-resposta ao tratamento com progestagênio é facilmente identificável pela sintomatologia (sangramento anormal) e pelo rastreamento histopatológico do endométrio.

O tratamento das hiperplasias de endométrio com AMP ou com AM representa alternativa satisfatória para mulheres que desejam preservar o útero ou que tenham risco cirúrgico elevado. Entretanto, é necessário monitorização cuidadosa do endométrio, o que pode ser realizado pela avaliação dos sintomas, pela ultra-sonografia transvaginal e pela biópsia periódica.

\section{SUMMARY}

Purpose: to evaluate the efficacy of medroxyprogesterone acetate and megestrol acetate in endometrial hyperplasia. Patients and Methods: forty-seven patients with abnormal uterine bleeding were retrospectively evaluated. These patients were submitted to diagnostic uterine curettage and/ or endometrial biopsy, with histopathological finding of endometrial hyperplasia. Patients with hyperplasia without 
atypia received $10 \mathrm{mg} /$ day oral medroxyprogesterone acetate during 10 to 12 days a month. Those with hyperplasia with atypia received $160 \mathrm{mg} /$ day oral megestrol acetate continuously. The length of treatment ranged from 3 to 18 months. Control endometrial biopsy and/or uterine curettage were performed 3 and 6 months from the beginning of treatment, and then periodically to evaluate whether or not regression of hyperplasia occurred.

Results: forty-two patients with endometrial hyperplasia without atypia and 5 with hyperplasia with atypia were included. The mean age of the patients was $49.5 \pm 10.6$ years (22 to 72 years), $70.2 \%$ aged over 45 years. Medroxyprogesterone acetate was effective in promoting regression of $83.2 \%$ (35/42) of hyperplasia without atypia, and megestrol acetate in 80\% (4/5) of hyperplasia with atypia. Despite treatment, lesions persisted in 16.8\% (7 cases) of hyperplasia with atypia and in 20\% (1 case) of hyperplasia without atypia. No progression to endometrial cancer was seen during the follow-up period of 3 months to 9 years. During follow-up, we found that 18 patients (38.3\%) showed amenorrhea, 12 (25.5\%) menstrual cycle regulation, and 17 (36.2\%) persistent abnormal uterine bleeding and underwent total abdominal hysterectomy. Histological examination of the uterus showed 8 patients with persistence of hyperplastic lesion, 4 with leiomyoma, 3 with adenomyosis, 1 with diffuse uterine myohypertrophy, and 1 with normal uterus, despite regression of the hyperplastic lesions in 9 of the 17 patients.

Conclusions: the treatment of endometrial hyperplasia with medroxyprogesterone acetate and megestrol acetate can be a safe alternative for women who refuse to have their uterus removed or those at high risk for surgery. However, a careful monitoring of the endometrium is needed. This can be achieved with periodical endometrial biopsy, transvaginal ultrasonography, and evaluation of the symptoms.

KEY WORDS: Endometrial hyperplasia. Medroxyprogesterone acetate. Endometrium: neoplasms. Uterine bleeding, abnormal.

\section{Referências}

1. Kurman RJ, Norris HJ. Endometrial hyperplasia and related cellular changes. In: Kurman RJ, editor. Blaustein's Pathology of the Female Genital Tract. $4^{\mathrm{a}}$ ed. New York: Springer-Verlag; 1994. p. 411-37.

2. Adler LM, Mutch DG. Endometrial hyperplasia. Infert Reprod Med 1995; 6:441-51.

3. Gusberg SB, Chen SY, Cohen CJ. Endometrial cancer: Factors influencing the choice of treatment. Gynecol Oncol 1974; 2:308-13.

4. Vellios F. Endometrial hyperplasia and carcinoma in situ. Gynecol Oncol 1974; 2:152-61.

5. Kurman RJ, Kamiski PF, Norris HJ. The behavior of endometrial hyperplasia. A long term study of "untreated" hyperplasia in 170 patients. Cancer 1985; 56:403-12.

6. Ferenczy A, Gelfand M, Tzipris F. The cytodynamics of endometrial hyperplasia and carcinoma. A review. Ann Pathol 1983; 3:189-91.

7. Creasy GW, Kafrissen ME, Upmalis D. Review of the endometrial effects of estrogens and progestins. Obstet Gynecol Surg 1992; 47:654-78.

8. Wentz WB. Treatment of persistent endometrial hyperplasia with progestagen. Am J Obstet Gynecol 1966; 96:999-1004.

9. Eichner E, Abellera M. Endometrial hyperplasia treated by progestagens. Obstet Gynecol 1971; 38:739-42.

10.Gal D, Edman CD, Vellios F, Forney JP. Long-term effect of megestrol acetate in the treatment of endometrial hyperplasia. Am J Obstet Gynecol 1983; 146:316-22.

11.Ferenczy A, Gelfand M. The biologic significance of cytologic atypia in progestogen treated endometrial hyperplasia. Am J Obstet Gynecol 1989; 160:126-31.

12.Gurpide E, Gusberg S, Tseng L. Estradiol binding and metabolism in human endometrial hyperplasia and adenocarcinoma. J Steroid Biochem 1976; 7:891.

13.Kirland JL, Murthy L, Stancel GM. Progesterone inhibits the estrogen induced expression of Cfos-messenger ribonucleic acid in the uterus. Endocrinology 1992; 130:3223-30.

14.Song JY, Fraser IS. Effects of progestogens on human endometrium. Obstet Gynecol Surv 1995; 50:385-94.

15.Kopelman PG. Investigation of obesity. Clin Endocrinol 1994; 41:703-8.

16. American Diabetes Association. Report of the Expert Committee on the diagnosis and classification of diabetes mellitus. Diabetes Care 1997; 20:1183-97.

17.Wentz WB. Progestin therapy in endometrial hyperplasia. Gynecol Oncol 1974; 2:362-7.

18.Taddei GL, Bargelli G, Scarselli B, Moncini D, Scarselli G. Precancerous lesions of the endometrium and medical treatment. Eur J Contracept Reprod Health Care 1997; 2:239-41.

19.Grabert H, Lima GR, France LC, Vivas AD, Miyada CC. Endometrial adenomatous hyperplasia analysis of the karyometric variations caused by medroxyprogesterone acetate. Rev Assoc Med Bras 1976; 22:147-53.

20.Randall TC, Kurman RJ. Progestin treatment of atypical hyperplasia and well diferenttiated carcinoma of the endometrium in women under age 40. Obstet Gynecol 1997; 90:434-40.

21.Burke TW, Tortolero-Luna T, Malpica A, et al. Endometrial hyperplasia and endometrial cancer. Obstet Gynecol Clin North Am 1996; 23:411-57.

22.Rodrigues de Lima G, Baracat EC, Girão MJBC. Aspectos endocrinos do carcinoma do endométrio. In: Rodrigues de Lima G, Baracat EC, editores. Ginecologia Endócrina. $1^{a}$ ed. São Paulo: Atheneu; 1995. p. 215-6.

23.Terakawa $\mathrm{N}$, Kigawa $\mathrm{J}$, Taketani $\mathrm{Y}$, et al. The behavior of endometrial hyperplasia a prospective study. Endometrial hyperplasia study group. J Obstet Gynecol Res 1997; 23:223-30.

24. Whitehead MI. Progestins and androgens. Fertil Steril 1994; 62(Suppl. 2):161-7. 